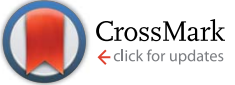

Cite this: RSC Adv., 2015, 5, 13762

Received 11th December 2014 Accepted 19th January 2015

DOI: 10.1039/c4ra16163g

www.rsc.org/advances

\section{Raman-encoded microbeads for spectral multiplexing with SERS detection $\uparrow$}

\begin{abstract}
Yuming Lai, abd Shuqing Sun, ${ }^{c}$ Tao He, ${ }^{d}$ Sebastian Schlücker ${ }^{a}$ and Yuling Wang ${ }^{\star a}$
Simultaneous detection of multiple molecular targets can greatly facilitate early diagnosis and drug discovery. Encoding micron-sized beads with optically active tags is one of the most popular methods to achieve multiplexing. Noble metal nanoparticle labels for optical detection by surface-enhanced Raman spectroscopy (SERS) exhibit narrow bandwidths, high photostability and intense Raman signals. In this study, we demonstrate the feasibility of spectral multiplexing by SERS using micron-sized polystyrene (PS) beads loaded with SERS-active nanoparticles. The silica-encapsulated SERS nanotags comprise gold nanocrystals with a self-assembled monolayer (SAM) of aromatic thiols as Raman reporter molecules for spectral identification. SERS microspectroscopic images of single Raman-encoded PS microbeads indicate the homogeneous spatial distribution of the SERS-active nanoparticles on the surface of the beads. By using up to five different Raman reporters, 31 spectrally distinct micron-sized beads were encoded and characterized spectroscopically at the single-bead level.
\end{abstract}

\section{Introduction}

Multiplexed identification of target molecules is particularly crucial for genomics and proteomics, ${ }^{1-3}$ medical diagnosis ${ }^{4}$ and drug discovery, ${ }^{5}$ which demand high throughput analysis of a large amount of biomolecules in a single reaction vessel. Traditional methods for multiplexed detection include multiplex ELISA (enzyme-linked immunosorbant assay) ${ }^{6,7}$ and microarrays, ${ }^{8,9}$ which actually suffer from some major drawbacks in data and array reproducibility, slow reaction kinetics due to the planar arrangement of probes, high cost and poor flexibility. ${ }^{10}$ Encoding strategies based on micron-sized beads have been emerging as attractive alternatives to current techniques for multiplexed detection schemes owing to their great advantages including enhanced reaction kinetics (suspension in homogenous solution), rapidity (shorter incubation and array times, faster analysis), simplicity of use, low cost, higher reproducibility and reliability. ${ }^{\mathbf{1 1}}$ Typically, this technology is based on encoding the microbeads with a unique identifier. A variety of techniques have been proposed and addressed for

${ }^{a}$ Faculty of Chemistry, University of Duisburg-Essen, D-45141, Germany. E-mail: yuling.wang@uni-due.de

${ }^{b}$ National Center for Materials Service Safety, University of Science and Technology Beijing, Beijing 100083, China

${ }^{c}$ Laboratory of Optical Imaging and Imaging, Shenzhen Key Laboratory for Minimally Invasive Medical Technologies, Graduate School at Shenzhen, Tsinghua University, Shenzhen, 518055, China

${ }^{d}$ Laboratory of Nanodevice, National Center for Nanoscience and Technology, Beijing, 100190, China

$\dagger$ Electronic supplementary information (ESI) available. See DOI: 10.1039/c4ra16163g encoding. Among these, encoding by quantum dots (QDs) ${ }^{\mathbf{1 2 , 1 3}}$ and molecular fluorophores/dyes ${ }^{\mathbf{1 4 , 1 5}}$ are most frequently used. Self-encoding with polymer particles as beads was also proposed by Fenniri's group, using FT-IR or Raman spectroscopy for read-out. ${ }^{16,17}$

Surface-enhanced Raman spectroscopy (SERS) ${ }^{18}$ has recently attracted great attention due to its high sensitivity, high photostability, and more importantly, the large multiplexing capability because of the narrow bands/fingerprint of the Raman molecules. ${ }^{19-23}$ Jun et al. have recently proposed an approach to encode beads by in situ growth of silver nanoparticles as SERS enhancer, followed by Raman reporters encoding, which shows the great potential by using SERS labels as tags for encoding of microbeads. ${ }^{24}$ However, the co-adsorption of Raman reporter molecules with $\mathrm{SiO}_{2}$ precursors (3-mercaptopropyltrimethoxysilane), leading to a sub-monolayer coverage of Raman reporter molecules on the surface, will lower the SERS intensity. On the other hand, the in situ growth of silver nanoparticles will generate different silver nanostructures, thus leading to poor reproducibility for the Raman enhancement. To improve the reproducibility and obtain maximum SERS signals, Schlücker and co-workers have introduced silica-encapsulated SERS labels with a complete self-assembled monolayer (SAM) of Raman reporters on the surface of noble metal nanoparticles (NPs) including gold $(\mathrm{Au}){ }^{25-28}$ The maximum surface coverage with Raman reporters on the surface of the AuNPs leads to maximum SERS signals and good reproducibility, while the silica surface enables further chemical modifications for bioconjugation.

Hence, a more general strategy was proposed to address the feasibility for a multiplexed SERS detection by using five different types of silica-encapsulated SAM-based SERS labels 
(AuNPs-Ra@SiO ${ }_{2}$ ) for encoding microbeads. Monodisperse AuNPs-Ra@SiO ${ }_{2}$ were first synthesized based on approaches reported earlier. ${ }^{25-28}$ SERS images of PS beads encoded by different SERS labels validate the multiplexing capability of this approach. With five types of SERS labels, all possible combinations (31 different codes) were obtained. We employ a binary notation for characterizing these combinations, where " 1 " represents the presence and " 0 " the absence of the corresponding SERS "colour", respectively. We expect to have five kinds of spectrally distinct codes with one type of SERS label (10000, 01000, 00100, 00010, 00001), 10 different codes with two types of SERS labels $(11000,10100,10010,10001,01100,01010$, 01001, 00110, 00101, 00011), 10 different codes with three types of SERS labels $(11100,11010,10110,01110,11001,10101$, 01101, 10011, 01011, 00111), five different codes with four different SERS labels (01111, 10111, 11011, 11101, 11110), and one code with all five SERS labels (11111).

\section{Experimental}

\section{Chemicals}

4-Mercaptobenzoic acid (MBA), 5,5'-dithiobis(2-nitrobenzoic) (DTNB), 2,7-mercapto-4-methylcoumarin (MMC), 2-mercapto-4methyl-5-thiazoleacetic acid (MMTAA), ethanol, isopropanol, tetraethoxyorthosilicate (TEOS), (3-aminopropyl)-trimethoxysilane (APTMS), ammonium hydroxide (30\%), $N^{\prime}$-(ethylcarbonimidoyl)$N, N$-dimethyl-monohydrochloride $\quad$ EDC, $N$-hydroxysulfosuccinimide sodium salt (s-NHS) and 4-(2-hydroxyethyl)-1-piperazineethanesulfonic acid (HEPES), were purchased from SigmaAldrich/Fluka. 2,3,5,6-Tetrafluoro-4-mercaptobenzoic acid (TFMBA) was purchased from Tokyo Chemical Industry (TCI) Co. MNBA was prepared by breaking the disulfide bond in DTNB with $\mathrm{NaBH}_{4}$ as reducing agent before use. Monodisperse polystyrene beads (PS) that contain surface carboxyl groups (PS-COOH) were purchased from micromod Partikeltechnologie GmbH. Ultrapure water $(18.2 \mathrm{M} \mathrm{cm})$ was used preparing all aqueous solution.

Monodispersed gold nanoparticles (AuNPs) with a diameter of around $70 \mathrm{~nm}$ were prepared through the procedure reported by Bastús et al. ${ }^{29}$

\section{Preparation of SERS nanoparticle labels}

Silica-encapsulated SAM-based SERS labels were obtained by encapsulation of Raman reporter-coated AuNPs (AuNPs-Ra) with a silica shell. ${ }^{25,27,28}$ Briefly, $300 \mathrm{~mL}$ colloidal AuNPs with a diameter of about $70 \mathrm{~nm}\left(\lambda_{\max }=548 \mathrm{~nm}\right)$ were centrifuged and redispersed in $100 \mathrm{~mL}$ ethanol. The colloid was then incubated overnight with $12 \mathrm{~mL}$ Raman reporter solutions $(10 \mathrm{mM}$ in ethanol) to form a complete self-assembled monolayer (SAM) on the particle surface. In this study, Raman reporters including MBA, MNBA, TFMBA, MMC and MMTAA (see Materials and methods for the chemical names of the aromatic thiols) were employed as Raman reporter in this proof-of-concept study. SAM-coated AuNPs were centrifuged and redispersed in ethanol $(50 \mathrm{~mL})$ to remove the excess Raman reporter molecules. For direct silica encapsulation of the SAM-coated gold nanoparticles (AuNPs-MBA), AuNPs were redispersed in a mixture of isopropanol (45 mL), water $(15 \mathrm{~mL})$, and ammonium hydroxide (1.6 mL). TEOS ( 1 vol\% in isopropanol) was then added under vigorous stirring in six portions over $3 \mathrm{~h}$. The thickness of the silica shell was adjusted by the amount of TEOS. For the silica encapsulation of the AuNPs-MNBA, AuNPs-TFMBA, AuNPsMMC, and AuNPs-MMTAA, APTMS is required before the Stöber synthesis with TEOS. Briefly, $2.7 \mathrm{~mL} 1 \mathrm{vol} \%$ APTMS (in ethanol) were added to the SAM-coated AuNPs. After shaking for 5 min, the colloidal dispersion was centrifuged and redispersed in a mixture of isopropanol $(45 \mathrm{~mL})$, water $(15 \mathrm{~mL})$, and ammonium hydroxide $(1.6 \mathrm{~mL})$. TEOS ( $1 \mathrm{vol} \%$ in isopropanol) was then added under vigorous stirring in six portions over $3 \mathrm{~h}$. The silica-encapsulated SERS labels were centrifuged after stirring overnight to remove excess ammonia and redispersed in $50 \mathrm{~mL}$ ethanol. The silica shell was hardened by heating at 65 ${ }^{\circ} \mathrm{C}$ for $5 \mathrm{~h}$ in ethanol with $750 \mathrm{rpm}$ shaking, which will greatly increase the stability of AuNPs-Ra@SiO ${ }_{2}$ according to the report. $^{30}$

\section{Conjugation of SERS NP labels onto the microbead surface}

Polystyrene (PS) beads were encoded by SERS NP labels via standard EDC conjugation chemistry. Briefly, silicaencapsulated SERS labels were activated by APTMS to obtain amino-functionalized SERS labels. In a typical experiment, 400 $\mu \mathrm{L}$ SERS label colloid (e.g. AuNPs-TFMBA@SiO ${ }_{2}$ ) was washed by ethanol for one more time to remove excess water and then 13 $\mu \mathrm{L} 0.5 \%$ APTMS together with $13 \mu \mathrm{L}$ ammonia were added to the SERS colloid under sonication for $5 \mathrm{~min}$ and shaking at room temperature. Finally, amino-functionalized SERS labels were washed and redispersed in $400 \mu \mathrm{L}$ HEPES ( $\mathrm{pH}$ 5.8) for further conjugation.

Monodisperse polystyrene (PS) beads containing surface carboxyl groups were activated by EDC/s-NHS. Briefly, $1.2 \mathrm{~mL}$ $0.5 \mathrm{mg} \mathrm{mL}{ }^{-1} \mathrm{PS}-\mathrm{COOH}$ was centrifuged and redispersed in HEPES buffer (pH 5.8) for activation. $450 \mu \mathrm{L} \mathrm{s}-\mathrm{NHS}$ (3 mg in 1.5 $\mathrm{mL}$ HEPES buffer) and $450 \mu \mathrm{L}$ EDC $(5 \mathrm{mg}$ in $1.5 \mathrm{~mL}$ HEPES buffer) were added into PS-COOH solution and shaked for 20 min to let s-NHS react with carboxyl groups. Then the solution with the PS beads was washed once in HEPES to remove access s-NHS/EDC and redispersed in HEPES. Subsequently, $100 \mu \mathrm{L}$ amino-functionalized silica-encapsulated SERS labels were added into $50 \mu \mathrm{L}$ activated PS beads solution under shaking for $1 \mathrm{~h}$. The total volume of SERS labels was kept constant, but the corresponding volume for each type of SERS labels varied, depending on the types of SERS labels encoded on the PS bead. Afterwards, the spectrally encoded micron-sized beads were washed and redispersed in ethanol for SERS microspectroscopic measurements.

\section{Instrumentation}

Extinction spectra of AuNPs and AuNPs-Ra@SiO ${ }_{2}$ were recorded with a Perkin-Elmer Lambda 35 UV-vis absorption spectrometer. Transmission electron microscopy (TEM) images were obtained with a Zeiss EM 902 instrument. Scanning electron microscopy (SEM) images were performed with a FEI Quanta 3D FEG 200/600 scanning electron microscope. 
Raman experiments were carried out with a WITec alpha 300 $\mathrm{R}$ Raman microscope. The $632.8 \mathrm{~nm}$ line from a He-Ne laser was used for excitation of Raman scattering. The monochromator comprises a 1800 grooves per $\mathrm{mm}$ grating. The acquisition time per Raman spectrum was $0.5 \mathrm{~s}$. The integrated Raman intensity (20 $\mathrm{cm}^{-1}$ "filters") around the dominant Raman shift of the corresponding Raman reporter molecule was calculated for generating the Raman false colour images. Average spectra were calculated by WITec project software.

\section{Result and discussion}

\section{Characterization of SERS labels and PS beads encoded with SERS labels}

Five different types of silica-encapsulated SERS labels were synthesized by using five different aryl thiols and characterized by TEM and extinction spectroscopy. Typical TEM images of silica-encapsulated SERS labels with TFMBA as the Raman reporter are displayed in Fig. 1a. Clearly, monodisperse silicaencapsulated AuNPs are observed and the shell thickness is adjusted to be around $12 \mathrm{~nm}$ by controlling the amount of TEOS. Silica-encapsulated AuNPs-Ra as SERS labels will therefore ensure the stability of the labels, prevent the interface from the environment and offer a versatile way for surface functionalization. Optical properties of AuNPs and AuNPs-Ra@SiO were also investigated by extinction spectroscopy (Fig. S1†), in which the distinct peak located at $548 \mathrm{~nm}$ is due to the localized surface plasmon resonance (LSPR) of AuNPs. After the formation of the SAM and the encapsulation by silica, the LSPR of AuNPs was red-shifted to $560 \mathrm{~nm}$ owing to the different environmental refractive index. It should be pointed out that the SERS labels are quite stable in water and in ethanol or isopropanol after hardening the silica shell. ${ }^{30}$

Furthermore, silica-encapsulated AuNPs-Ra were modified by a silane agent (APTMS) to introduce amino groups on the silica surface, which will react with activated carboxyl groups on the surface of the PS beads $(\sim 5 \mu \mathrm{m})$. The ratio of SERS labels to PS beads needs to be carefully adjusted. To validate the binding between SERS labels and the PS bead, scanning electron microscopy (SEM) was employed to characterize the SERS labels on the PS beads surface after the reaction as indicated in

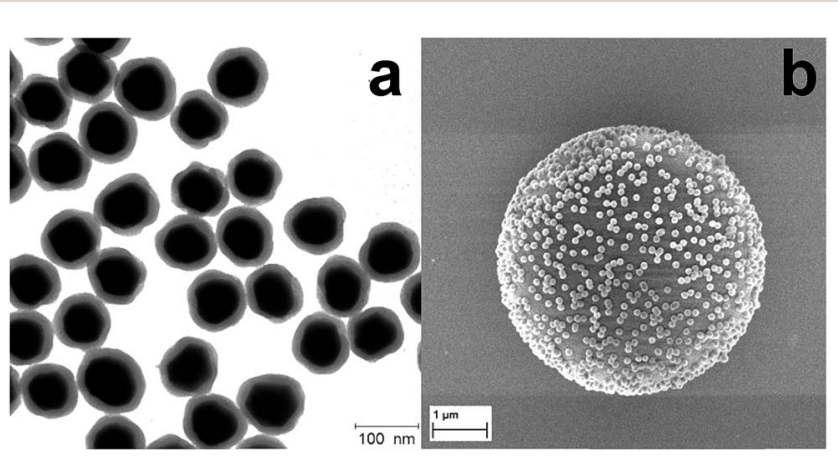

Fig. 1 (a) Typical TEM image of SERS labels (AuNPs-TFMBACSiO 2 ) and (b) SEM image of a single PS bead encoded with SERS nanoparticle labels.
Fig. 1b: a monolayer of SERS labels was observed clearly on the single PS bead surface. No aggregation or multilayer formation was observed, indicating the strong covalent binding of SERS labels with PS beads.

SERS labels with distinct spectroscopic peaks are needed to achieve multiple spectrally encoded micron-sized beads for optical readout by SERS. As discussed, the small size of aromatic molecules with the uniform orientation on AuNPs surface will enable the multiplexed detection owing to the appearance of only few distinct vibrational Raman peaks. Normalized SERS spectra and the corresponding molecular structures from five types of SERS labels including MBA, MMC, MMTAA, MNBA and TFMBA were obtained as depicted in Fig. 2. Distinct peaks at $1080 \mathrm{~cm}^{-1}, 1172 \mathrm{~cm}^{-1}, 1290 \mathrm{~cm}^{-1}, 1337 \mathrm{~cm}^{-1}$ and $1380 \mathrm{~cm}^{-1}$ were observed, which are characteristic of their own SERS signatures, ensuring an unambiguous spectral identification. The SERS spectrum of each type of SERS label can be easily distinguished because the most intensive peak of each Raman reporter molecule is separated by at least $35 \mathrm{~cm}^{-1}$. Although a mixture of these 5 different SERS labels can also display spectral barcodes, it only shows 5 barcodes, while encoding on PS beads, 31 spectrally distinct SERS signatures (barcodes) can be obtained by mixing up to 5 different Raman reporters.

\section{Raman mapping of SERS NP-encoded PS beads}

The micron-sized PS beads can be easily identified by optical microscopy and then characterized by Raman microscopy. As indicated in Fig. 3, SERS label-encoded PS beads were analysed by SERS microspectroscopy. Fig. 3a shows the results obtained with one type of SERS label encoding the PS bead (binary code: 00010). The false-colour SERS image on a single PS bead was achieved by integrating the Raman intensity between 1320 and $1360 \mathrm{~cm}^{-1}$. It was found that optical and Raman false-colour images showed good correspondence and, importantly, a homogenous distribution of SERS signals across the entire PS bead, demonstrating the uniform coverage of SERS labels on the bead surface. This is very important for SERS applications in multiplexed detection to ensure the reproducibility, reliability and low-cost because the measurement can be performed on a

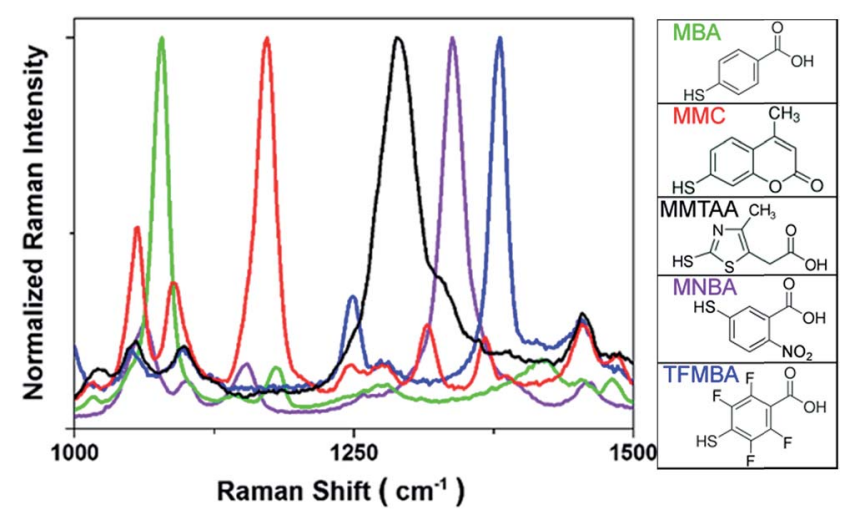

Fig. 2 SERS spectra of AuNPs-Ra@ $\mathrm{SiO}_{2}$ (left) and molecular structures of Raman reporters employed in this study (right). 


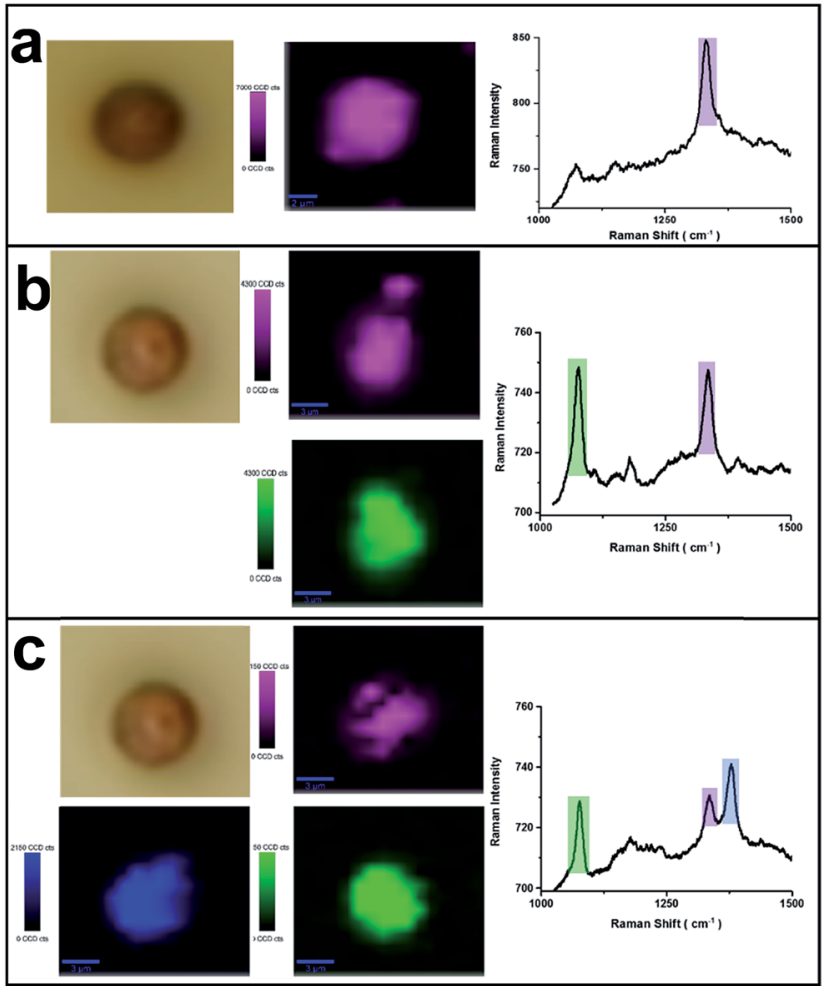

Fig. 3 Optical and false-colour SERS images of a single PS bead with one type of SERS label ((a) 00010), two types of SERS labels ((b) 10010), and three types of SERS labels ((c) 10011). Average Raman spectrum from SERS labels encoded on an individual PS bead with MNBA (1337 $\mathrm{cm}^{-1}$ ) (purple); MBA $\left(1080 \mathrm{~cm}^{-1}\right.$ ) (green) and TFMBA $\left(1380 \mathrm{~cm}^{-1}\right.$ ) (blue).

single bead. The average Raman spectrum of the whole bead with SERS labels (MNBA) is shown in the right of Fig. 3a, exhibiting the characteristic peak of the symmetric nitro stretching vibration at $1337 \mathrm{~cm}^{-1}$.

SERS images from other types of SERS label-encoded PS beads were characterized accordingly. The corresponding Raman false colours are shown in Fig. $3 \mathrm{~b}$ and c, representing the combinations 10010 and 10011, respectively. Notably, the average SERS intensity on each type of SERS label-encoded PS bead varies. Typically, the Raman intensity of the characteristic peak from one SERS label decreased with increasing amounts of other SERS labels on the same bead. For instance, the Raman intensity of the characteristic nitro peak at 1337 $\mathrm{cm}^{-1}$ from only one type of SERS label (00010) was $c a .80$ counts, while the Raman intensity of the same peak decreased to $c a$. 40 counts and 20 counts, respectively, in the case of the combination 10010 and 10011, in which two different SERS labels are present on the same PS bead. This can easily be explained by the constant total number of SERS nanoparticle labels on the PS bead surface. Because the encoding of SERS labels on PS bead surface is based on covalent binding and the formation of a monolayer of SERS nanoparticles (as illustrated in the SEM image of Fig. 1b), the total amount of SERS labels on one PS bead is nearly the same. These results further demonstrate the feasibility of spectroscopically encoded beads for multiplexed SERS detection. Overall, 31 different spectral barcodes by SERS label-encoded PS beads can theoretically be achieved (only binary notations, i.e., not including different intensity ratios).

\section{Spectral barcodes from SERS label-encoded PS beads}

Fig. 4 (left) shows the average Raman spectra from five SERS-NPcoated PS beads, each one spectrally encoded with a different Raman reporter (single "colour"), together with the average Raman spectrum from a PS bead encoded by a mixture of all 5 types of SERS NP labels (five "colours"). Each bead with one "colour" shows a characteristic peak and the bead encoded with five types of SERS labels can be distinguished clearly by 5 distinct peaks, which are from each type of SERS label. The corresponding barcode representations of the Raman spectra are shown in Fig. 4 (right). In the spectral barcode representation, each characteristic Raman peak is represented by a vertical line in a different colour.

The other 25 SERS spectra and the corresponding barcodes, which were obtained experimentally by a combination of up to four types SERS labels, are shown in Fig. 5 and 6. Overall, we can differentiate 31 kinds of SERS label-encoded PS beads, which could be very useful in multiplexed identification using single beads.

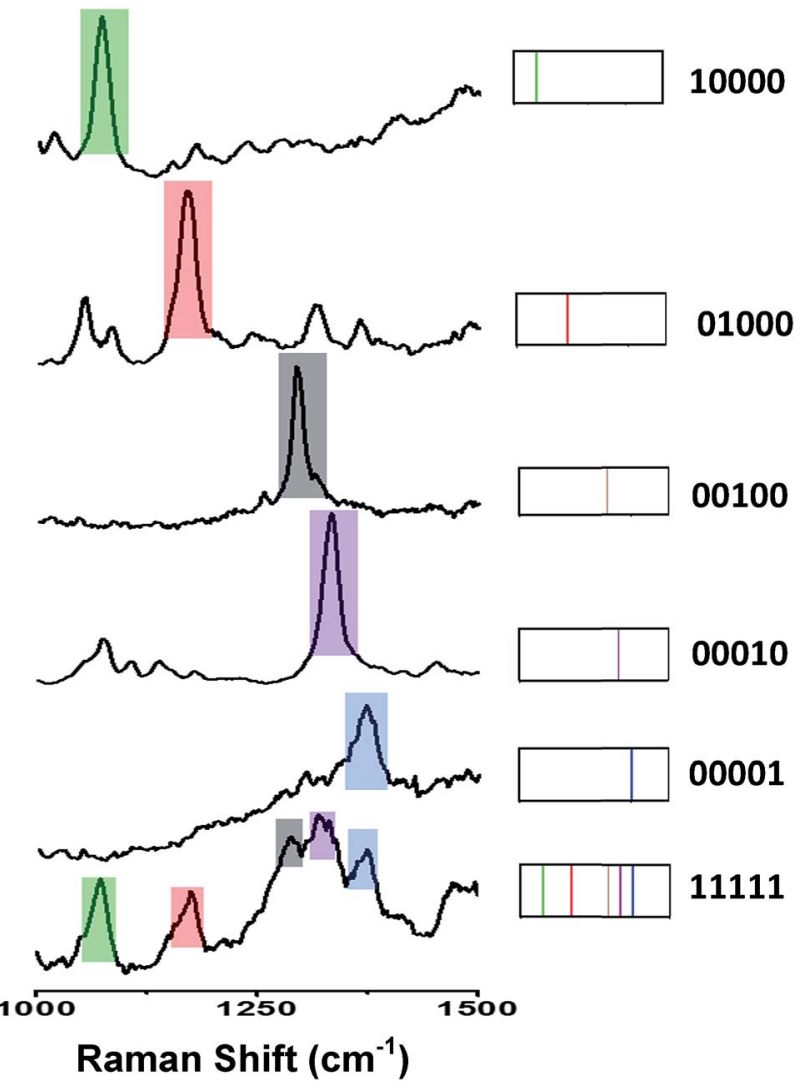

Fig. 4 Typical Raman spectra (left) on microbeads with different types of SERS label using different Raman reporters (10000, 01000, 00100 , 00010,00001 and 11111) and their corresponding spectral barcodes (right). 

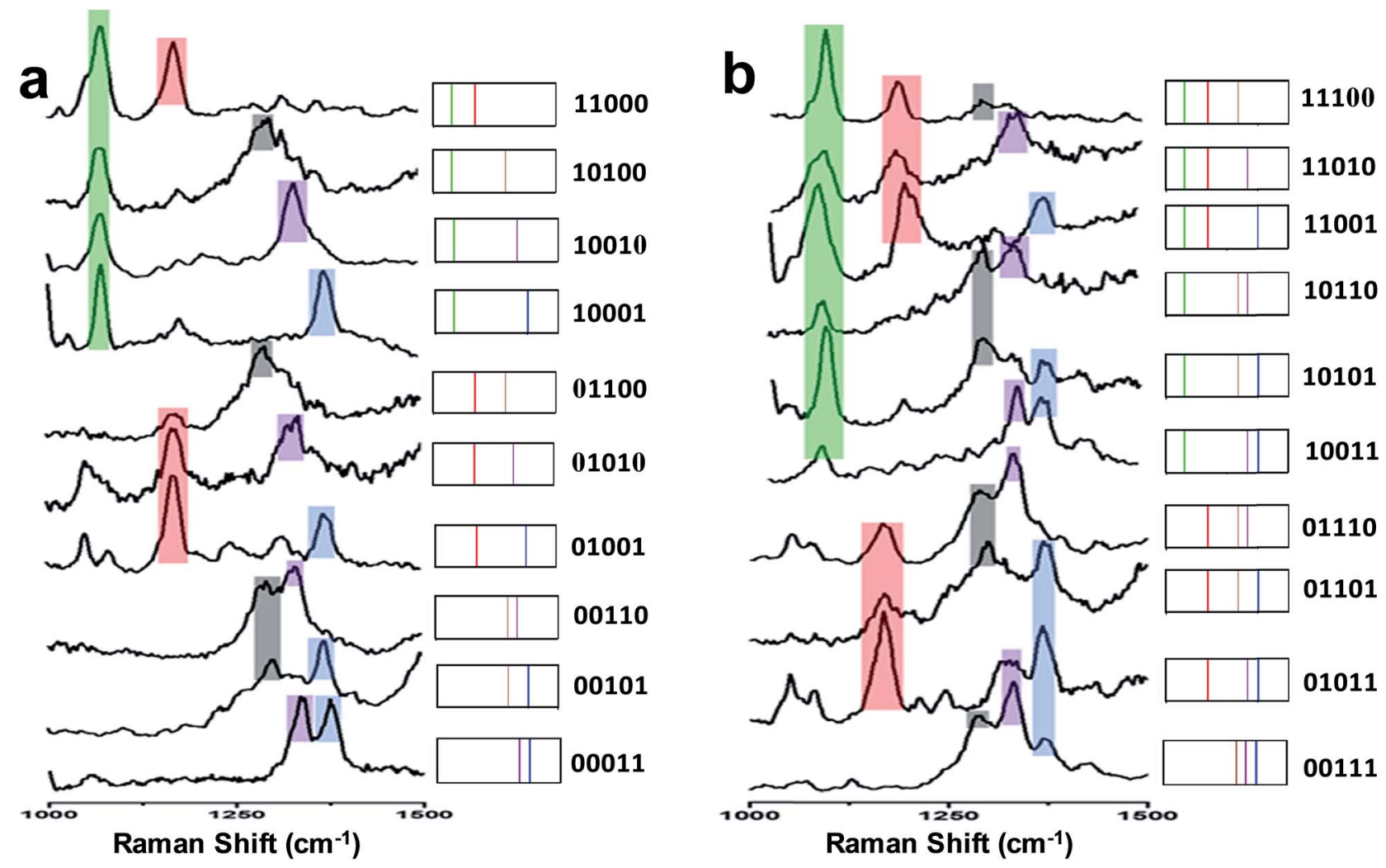

Fig. 5 (a) SERS spectra and barcode representation of two types SERS labels on PS bead. (b) SERS spectra and barcode representation of three type SERS labels on PS bead.

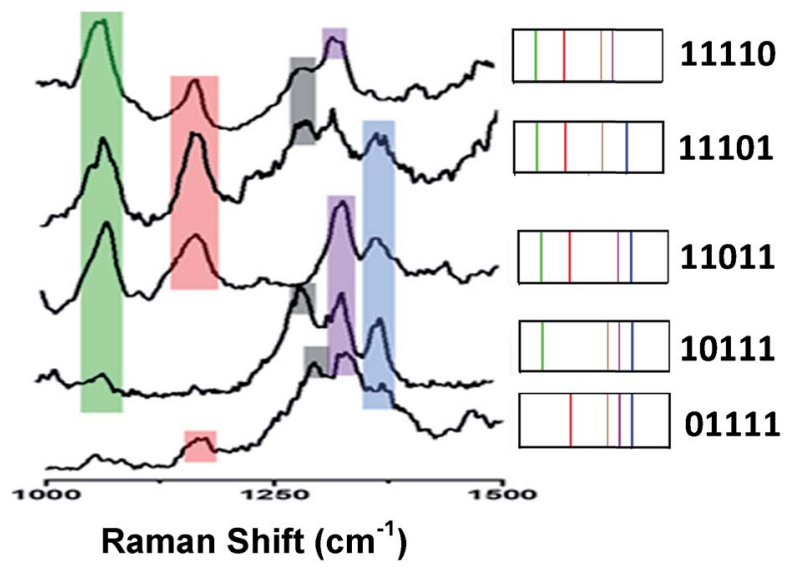

Fig. 6 Typical SERS spectra and barcode representation pictures of four type SERS labels on PS bead.

\section{Conclusions}

In summary, silica-encapsulated self-assembled monolayers of Raman reporter-coated AuNPs (AuNPs-Ra@SiO ${ }_{2}$ ) were synthesized and employed as bright and stable SERS labels for encoding single micron-sized PS beads for multiplexed optical detection. The particles are characterized by extinction spectroscopy, TEM and SEM. Single SERS label-encoded PS beads were further studied by SERS microspectroscopy. The SERS nanoparticles are homogeneously distributed across the single bead. Using up to 5 different Raman reporters, 31 distinct spectral barcodes from single micron-sized bead were obtained, demonstrating the great potential of this approach for future multiplexed biomarker detection schemes based on SERS.

\section{Acknowledgements}

Financial support from the German Research Foundation (DFG/ WA3369/1-1) is acknowledged. Y. M. Lai thanks the China Scholarship Council for the financial support of her research visit at the University of Duisburg-Essen and Fundamental Research Funds for the Central Universities (FRF-TP-14-058A1) for the financial support. T.H. would like to thank National Research Fund for Fundamental Key Projects no. 973 (2011CB933200) for the financial support.

\section{References}

1 G. A. Evans and K. A. Lewis, Proc. Natl. Acad. Sci. U. S. A., 1989, 86, 5030.

2 P. Ng, J. J. S. Tan, H. S. Ooi, Y. L. Lee, K. P. Chiu, M. J. Fullwood, K. G. Srinivasan, C. Perbost, L. Du, W. K. Sung, C. L. Wei and Y. Ruan, Nucleic Acids Res., 2006, 34, e84.

3 E. Boja, T. Hiltke, R. Rivers, C. Kinsinger, A. Rahbar, M. Mesri and H. Rodriguez, J. Proteome Res., 2011, 10, 66. 
4 D. A. A. Vignali, J. Immunol. Methods, 2000, 243, 243.

5 K. S. Lam, M. L. Lam and K. Viktor, Chem. Rev., 1997, 97, 411.

6 Quansys Bio multiplex ELISA kit specifications: http:// www.Quansysbio.com.

7 Ray Biotech multiplex ELISA kit specifications: http:// www.raybiotech.com.

8 G. L. G. Miklos and R. Maleszka, Nat. Biotechnol., 2004, 22, 615.

9 R. D. Canales, Y. Luo, J. C. Willey, B. Austermiller, C. C. Barbacioru, C. Boysen, K. Hunkapiller and R. V. Jensen, Nat. Biotechnol., 2006, 24, 1115.

10 S. Birtwell and H. Morgan, Integr. Biol., 2009, 1, 345.

11 B. Battersby and M. Trau, Aust. J. Chem., 2007, 60, 343.

12 L. C. Mattheakis, J. M. Dias, Y. Choi, J. Gong, M. P. Bruchez, J. Liu and E. Wang, Anal. Biochem., 2004, 327, 200.

13 Y. Zhao, H. C. Shum, H. Chen, L. L. A. Adams, Z. Gu and D. A. Weitz, J. Am. Chem. Soc., 2011, 133, 8790.

14 X. W. Zhao, Z. B. Liu, H. Yang, K. Nagai, Y. H. Zhao and Z.-Z. Gu, Chem. Mater., 2006, 18, 2443.

15 B. J. Battersby, D. Bryant, W. Meutermans, D. Matthews, M. L. Smythe and M. Trau, J. Am. Chem. Soc., 2000, 122, 2138.

16 H. Fenniri, O. Terreau, S. Chun, S. J. Oh, W. F. Finney and M. D. Morris, J. Comb. Chem., 2006, 8, 192.

17 J. Raez, D. R. Blais, Y. Zhang, R. A. Alvarez-Puebla, J. P. BravoVasquez, J. P. Pezacki and H. Fenniri, Langmuir, 2007, 23, 6482.
18 S. Schlücker, ChemPhysChem, 2009, 10, 1344.

19 S. Schlücker, Angew. Chem., Int. Ed., 2014, 53, 4756.

20 Y. L. Wang and S. Schlücker, Analyst, 2013, 138, 2224.

21 H. Qu, Y. M. Lai, D. Z. Niu and S. Q. Sun, Anal. Chim. Acta, 2013, 763, 38.

22 Y. M. Lai, F. Li and S. Q. Sun, Integr. Ferroelectr., 2013, 146, 88.

23 Y. M. Lai, J. Wang, T. He and S. Q. Sun, Anal. Lett., 2014, 47, 833.

24 B. H. Jun, J. H. Kim, H. Park, J. S. Kim, K. N. Yu, S. M. Lee, H. Choi, S. Y. Kwak, Y. K. Kim, D. H. Jeong, M. H. Cho and Y. S. Lee, J. Comb. Chem., 2007, 9, 237.

25 B. Küstner, M. Gellner, M. Schütz, F. Schöppler, A. Marx, P. Ströbel, P. Adam, C. Schmuck and S. Schlücker, Angew. Chem., Int. Ed., 2009, 48, 1950.

26 M. Gellner, K. Kömpe and S. Schlücker, Anal. Bioanal. Chem., 2009, 394, 1839.

27 M. Schütz, B. Küstner, M. Bauer, C. Schmuck and S. Schlücker, Small, 2010, 6, 733.

28 M. Schütz, M. Salehi and S. Schlücker, Chem.-Asian J., 2014, 9, 2219.

29 N. G. Bastús, J. Comenge and V. Puntes, Langmuir, 2011, 27, 11098.

30 Y. J. Wong, L. Zhu, W. S. Teo, Y. W. Tan, Y. Yang, C. Wang and H. Chen, J. Am. Chem. Soc., 2011, 133, 11422. 